\title{
Colloidal charge renormalization in suspensions containing multivalent electrolyte
}

Cite as: J. Chem. Phys. 132, 104105 (2010); https://doi.org/10.1063/1.3354120

Submitted: 10 December 2009 . Accepted: 14 February 2010. Published Online: 10 March 2010

Alexandre P. dos Santos, Alexandre Diehl, and Yan Levin

\section{ARTICLES YOU MAY BE INTERESTED IN}

Charge renormalization, osmotic pressure, and bulk modulus of colloidal crystals: Theory

The Journal of Chemical Physics 80, 5776 (1984); https://doi.org/10.1063/1.446600

Charge neutrality breakdown in confined aqueous electrolytes: Theory and simulation The Journal of Chemical Physics 145, 094704 (2016); https://doi.org/10.1063/1.4962198

Effects of the dielectric discontinuity on the counterion distribution in a colloidal suspension The Journal of Chemical Physics 135, 044124 (2011); https://doi.org/10.1063/1.3615940

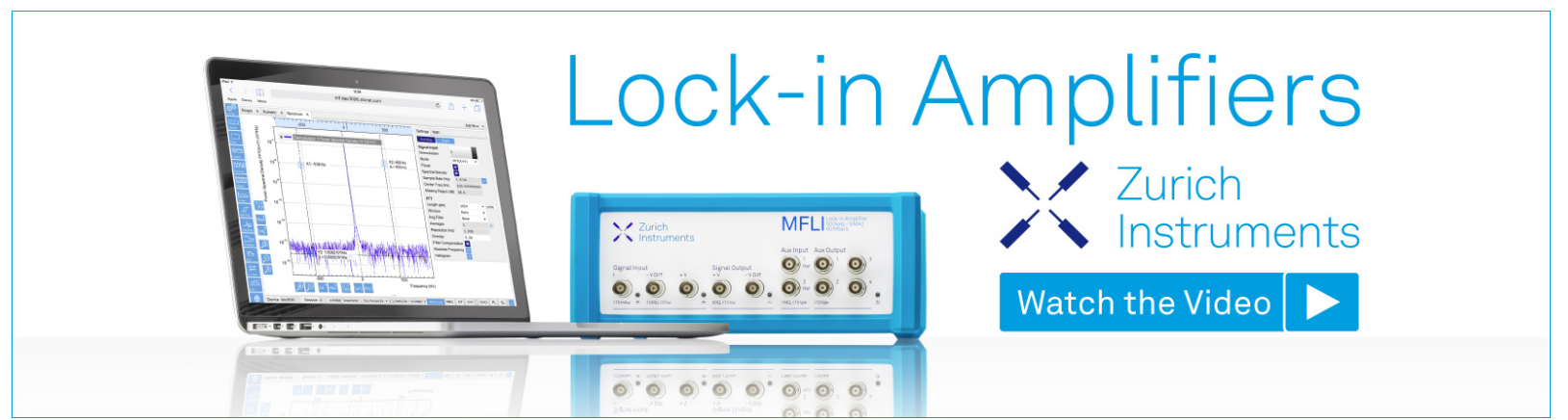




\title{
Colloidal charge renormalization in suspensions containing multivalent electrolyte
}

\author{
Alexandre P. dos Santos, ${ }^{1}$ Alexandre Diehl, ${ }^{2}$ and Yan Levin ${ }^{1, a)}$ \\ ${ }^{1}$ Instituto de Física, Universidade Federal do Rio Grande do Sul, Caixa Postal 15051, \\ CEP 91501-970 Porto Alegre, RS, Brazil \\ ${ }^{2}$ Departamento de Física, Instituto de Física e Matemática, Universidade Federal de Pelotas, \\ Caixa Postal 354, CEP 96010-900 Pelotas, RS, Brazil
}

(Received 10 December 2009; accepted 14 February 2010; published online 10 March 2010)

\begin{abstract}
A theory is proposed which allows us to self-consistently calculate the effective colloidal charge and the counterion and coion density profiles in suspensions containing both multivalent and monovalent electrolytes. The formation of counterion-coion clusters is explicitly taken into account. The theory predicts that sufficiently strongly charged colloidal particles will become overcharged. The addition of monovalent electrolyte decreases the counterion condensation and diminishes the amount of charge reversal. Predictions of the theory are compared with the Monte Carlo simulations and are found to be in excellent agreement without any adjustable parameters. () 2010 American Institute of Physics. [doi:10.1063/1.3354120]
\end{abstract}

\section{INTRODUCTION}

Quantitative theories for systems with long range interactions present an outstanding challenge to statistical mechanics. Even such basic question as the universality class of a symmetric two component plasma has been debated vigorously until very recently. ${ }^{1}$ Asymmetric systems, such as colloidal suspensions, are significantly more difficult. ${ }^{2}$

For aqueous colloidal suspensions containing only $1: 1$ electrolyte, Poisson-Boltzmann (PB) theory provides an accurate description of thermodynamic properties. ${ }^{3}$ It also gives a very simple picture of colloid-colloid interactions. Strong attraction between colloidal particles and counterions leads to formation of colloid-counterion complexes interacting by a screened Yukawa-like potential. The renormalized colloidal charge - the net charge of the complex — can be significantly lower than its "bare" chemical charge.

For suspensions containing multivalent ions, PB theory fails dramatically. In this case, strong ionic correlations between condensed counterions can lead to attraction between like-charged colloidal particles ${ }^{2,4}$ and charge reversal. ${ }^{5}$ Electrostatic correlations can also result in a reversal of the electrophoretic mobility. ${ }^{6}$ This means that more counterions condense onto colloidal particle than is necessary to completely neutralize its bare charge. This is in stark contrast to predictions of the PB theory, which finds that the effective colloidal charge saturates, but remains of the same sign as the bare charge. ${ }^{7}$

To calculate the effective charge within the PB theory one can use the, so called, Alexander prescription. ${ }^{7}$ A colloidal particle with its counterions and coions, is placed inside a spherical Wigner-Seitz (WS) cell. The numerical solution of the nonlinear $\mathrm{PB}$ equation is then asymptotically matched to the solution of the linearized $\mathrm{PB}$ equation with an effective colloidal charge. The prescription works very well for aque-

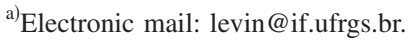

ous 1:1 electrolytes, for which the interaction between the counterions and coions is weak.

Unfortunately, there appears to be no direct way to extend the Alexander prescription to suspensions containing multivalent salts. ${ }^{8}$ The reason for this is that even the bulk thermodynamics of asymmetric multivalent electrolyte (such as $3: 1$ or $4: 1$ salts) is difficult to describe quantitatively. For these systems, one finds that cations and anions associate to form charged clusters, and the PB theory looses its validity. In this paper, we will present a new theory which will allow us to quantitatively calculate both the far-field ionic density profiles and the effective charges of colloidal particles, for suspensions containing multivalent electrolyte.

\section{THE MODEL}

To model a colloidal suspension containing $\alpha: 1$ electrolyte we will use the usual WS cell approach. Each colloidal particle of radius $a$ and charge $-Z q$, where $q$ is the proton charge, is placed at the center of a WS cell of radius $R$, determined from the colloidal concentration. The cell also contains $N_{\text {salt }}$ counterions ( $\alpha$-ions) of charge $+\alpha q$, and radius $r_{c}$; and $\alpha N_{\text {salt }}$ coions of charge $-q$ and radius $r_{-}$. In order to keep the electroneutrality, $Z$ monovalent counterions of charge $+q$ and radius $r_{+}$are also present within the cell. The solvent is treated as a structureless medium of dielectric permittivity $\epsilon$. To simplify notation, we will take all the ions to have the same radius $r_{+}=r_{-}=r_{c}$. In the rest of the paper, the colloidal radius will be fixed at $a=30 \AA$ and the ionic radius at $r_{c}=2 \AA$. The Bjerrum length $\lambda_{B}=q^{2} \beta / \epsilon$, where $\beta$ $=1 / k_{B} T$, will be fixed at $\lambda_{B}=7.2 \AA$, the value for water at room temperature.

For 1:1 salt, ionic charge distribution can be calculated accurately using the PB equation ${ }^{2}$ 


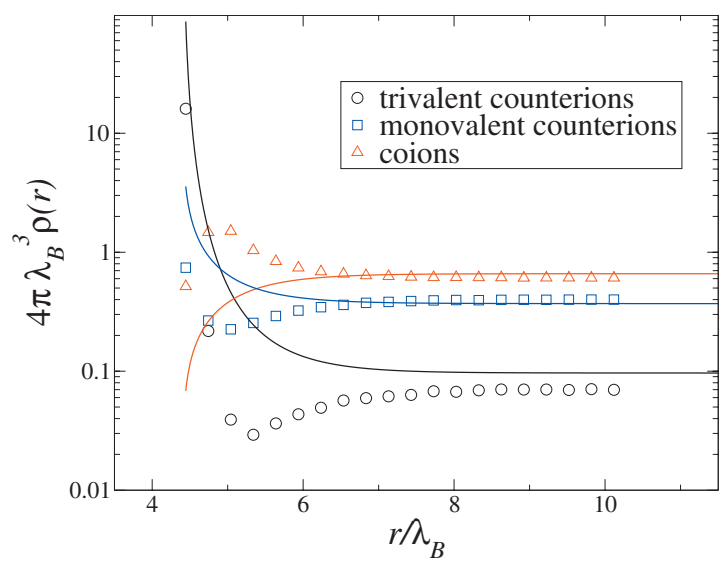

FIG. 1. Ionic density profiles for 3:1 electrolyte at concentration $0.075 \mathrm{M}$. The colloidal charge is $-270 q$. The WS cell radius is $93.05 \AA$. The symbols are the simulation data and the lines are the solution of the PB equation.

$$
\nabla^{2} \phi(r)=\frac{Z q}{\epsilon a^{2}} \delta(r-a)-\frac{4 \pi q}{\epsilon}\left[\rho_{+}(r)-\rho_{-}(r)\right]
$$

where $\phi(r)$ is the electrostatic potential at distance $r$ from the colloidal center, and the densities are

$$
\rho_{ \pm}(r)=\frac{N_{ \pm} \exp [\mp \beta q \phi(r)]}{4 \pi \int_{a}^{R} d r r^{2} \exp [\mp \beta q \phi(r)]},
$$

where $N_{ \pm}$are the number of \pm particles.

If suspension contains $\alpha: 1$ electrolyte, or if the dielectric constant is low, the correlations between cations and anions become important, and the PB equation looses its validity. In Fig. 1, we compare the density profiles of $3: 1$ electrolyte calculated using the $\mathrm{PB}$ equation to the results of the Monte Carlo (MC) simulations. The simulations were performed in the NVT ensemble using the Ewald summation method. ${ }^{9}$ It is clear that PB theory fails dramatically, even far from colloidal surface. An alternative to $\mathrm{PB}$ is to use integral equations $^{10-12}$ or weighted density functional theories, ${ }^{13-15}$ which, however, also lack in quantitative accuracy and are more difficult to solve numerically, than the simple PB equation.

\section{THE THEORY}

The failure of PB equation is a consequence of two effects: strong electrostatic correlations between the condensed counterions, and strong electrostatic correlations between the cations and anions of the bulk. Let us start with the ionic correlations in the bulk. In the rest of the paper, we fix $\alpha$ $=3$. Electrostatic attraction between trivalent counterions and monovalent coions results in their association. From simulations we observe that the dominant cluster consists of one monovalent coion associated with one trivalent counterion, forming a pair. To simplify the theory, we will neglect the formation of higher order clusters for now. The law of mass action relates the electrochemical potential of the cluster and of free ions

$$
\mu_{p}=\mu_{f}+\mu_{-} .
$$

The electrochemical potentials are given by

$$
\begin{aligned}
& \beta \mu_{p}=\ln \left[\frac{c_{p} \Lambda^{6}}{\xi_{p}}\right]+\beta \mu_{p}^{\mathrm{ex}}, \quad \beta \mu_{f}=\ln \left[c_{f} \Lambda^{3}\right]+\beta \mu_{f}^{\mathrm{ex}}, \\
& \beta \mu_{-}=\ln \left[c_{-} \Lambda^{3}\right]+\beta \mu_{-}^{\mathrm{ex}} .
\end{aligned}
$$

Substituting Eqs. (4) into Eq. (3), we can relate the concentration of cation-anion pairs $c_{p}$, to the concentrations of free $\alpha$-ions $c_{f}$, and to the concentration of monovalent coions $c_{-}$ (Ref. 16)

$$
c_{p}=\xi_{p} c_{f} c_{-} e^{-\beta\left(\mu_{p}^{\mathrm{ex}}-\mu_{f}^{\mathrm{ex}}-\mu_{-}^{\mathrm{ex}}\right)} .
$$

The internal partition function of a cluster is (Ref. 16)

$$
\xi_{p}=4 \pi \int_{r_{c}}^{R_{s}} e^{-\beta U(r)} r^{2} d r
$$

where $U(r)=-r_{c} b / r$ is the Coulomb potential, $b=\alpha \lambda_{B} / 2 r_{c}$ and $R_{s}=r_{c} b / 2$. Integrating we obtain

$$
\xi_{p}=\frac{16 \pi r_{c}}{3}\left[b^{3}\left(\operatorname{Ei}(b)-\operatorname{Ei}(2)+e^{2}\right)-e^{b}\left(b^{2}+b+2\right)\right],
$$

where $\operatorname{Ei}(x)$ is the exponential integral function. ${ }^{16}$ The excess chemical potentials of ions and clusters can be obtained using the Debye-Hückel theory. ${ }^{2}$ We find (Refs. 16 and 17)

$$
\begin{aligned}
& \beta \mu_{p}^{\mathrm{ex}}=-\frac{(\alpha-1)^{2} \lambda_{B} \kappa}{2\left(1+2 \kappa r_{p}\right)}, \quad \beta \mu_{f}^{\mathrm{ex}}=-\frac{\alpha^{2} \lambda_{B} \kappa}{2\left(1+2 \kappa r_{c}\right)}, \\
& \beta \mu_{-}^{\mathrm{ex}}=-\frac{\lambda_{B} \kappa}{2\left(1+2 \kappa r_{c}\right)}
\end{aligned}
$$

where $r_{p}=1.191 r_{c}, \kappa=\sqrt{8 \pi \lambda_{B} I}$ is the inverse Debye length and $I=\left[\alpha^{2} N_{f}+(\alpha-1)^{2} N_{p}+N_{-}+N_{+}\right] / 2 V$ is the ionic strength. $V$ is the volume accessible to ions, $N_{f}=c_{f} V, N_{p}=c_{p} V, N_{-}$ $=c_{-} V$, and $N_{+}=Z$. Note that $N_{\text {bulk }}=N_{\text {salt }}-N_{c}=N_{f}+N_{p}$, where $N_{c}$ is the number of condensed counterions, and $\alpha N_{\text {salt }}=N_{-}$ $+N_{p}$.

To account for electrostatic correlations between the condensed counterions, we shall adopt an argument proposed by Shklovskii. ${ }^{18}$ Shklovskii suggested that it might still be possible to use the PB theory, however, the boundary condition at the colloidal surface must be renormalized to account for the counterion condensation. Previously, we saw that because of cation-anion association, naive PB theory cannot be used to describe suspensions containing 3:1 electrolyte. However, the renormalized boundary condition can be implemented in a modified PB (mPB) equation that explicitly takes into account cluster formation,

$$
\nabla^{2} \phi(r)=-\frac{4 \pi q}{\epsilon}\left[\alpha \rho_{f}(r)+(\alpha-1) \rho_{p}(r)+\rho_{+}(r)-\rho_{-}(r)\right],
$$

where

$$
\rho_{f}(r)=\frac{N_{f} \exp [-\beta \alpha q \phi(r)]}{4 \pi \int_{a}^{R} d r r^{2} \exp [-\beta \alpha q \phi(r)]},
$$




$$
\rho_{p}(r)=\frac{N_{p} \exp [-\beta(\alpha-1) q \phi(r)]}{4 \pi \int_{a}^{R} d r r^{2} \exp [-\beta(\alpha-1) q \phi(r)]},
$$

and the densities of monovalent counterions and coions are given by Eq. (2). The zero of the electrostatic potential will be set at the boundary of the WS cell where the electric field also vanishes due to the charge neutrality. To obtain the boundary condition at the colloidal surface, we note that a strong attraction between the multivalent counterions and colloidal particles results in counterion condensation. The condensed $\alpha$-ions will form a quasi two dimensional strongly correlated fluid on top of colloidal surface. This fluid will be in thermodynamic equilibrium with the bulk electrolyte. The electrochemical potential of condensed counterions is

$$
\beta \mu_{\mathrm{sc}}=\beta \mu_{c}+\ln \left[\rho_{\mathrm{sc}} \Lambda^{3}\right]+\beta \alpha q \phi(a),
$$

where $\Lambda$ is the thermal de Broglie wavelength, $\rho_{\mathrm{sc}}$ is the average (coarse-grained) concentration of condensed counterions near the colloidal surface, $\alpha q \phi(a)$ is the mean electrostatic energy of bound counterions, and

$$
\beta \mu_{c}=-\left(1.65 \Gamma-2.61 \Gamma^{1 / 4}+0.26 \ln \Gamma+1.95\right)
$$

is their excess chemical potential, ${ }^{19}$ with plasma parameter $\Gamma=\alpha^{2} \lambda_{B} \sqrt{N_{c}} / 2\left(a+r_{c}\right)$. The electrochemical potential of diffuse layer counterions at distance $r$ from colloidal center is

$$
\beta \mu_{f}^{\text {bulk }}(r)=\ln \left[\rho_{f}(r) \Lambda^{3}\right]+\beta \alpha q \phi(r)+\beta \mu_{f}^{\mathrm{ex}} .
$$

We only need to know the excess electrochemical potential $\mu_{f}^{\mathrm{ex}}$, since predominantly free $\alpha$-ions will condense from the bulk. Condition of thermodynamic equilibrium then relates the density of free $\alpha$-ions in the bulk with the coarse-grained counterion density $\rho_{\text {sc }}$ near the colloidal surface,

$$
\rho_{f}(r)=\rho_{\mathrm{sc}} e^{\beta\left(\mu_{c}+q \alpha[\phi(0)-\phi(r)]-\mu_{f}^{\mathrm{ex}}\right)} .
$$

Near the surface, variation of the mean electrostatic energy is much smaller than $\mu_{c}$, so that Eq. (15) simplifies to

$$
\rho_{f}(a+\delta)=\rho_{\mathrm{sc}} e^{\beta\left(\mu_{c}-\mu_{f}^{\mathrm{ex}}\right)}
$$

which can be extrapolated all the way to the colloidal surface, $\delta \rightarrow r_{c}$. A scaling argument ${ }^{20,21}$ suggests that

$$
\rho_{\mathrm{sc}}=N_{c} / 4 \pi a^{2} \lambda_{G C}
$$

where $\lambda_{G C}=2\left(a+r_{c}\right)^{2} / Z \alpha \lambda_{B}$ is the Gouy-Chapman length. The concentration of trivalent electrolyte in the diffuse layer, extrapolated to colloidal surface is then

$$
\rho_{f}^{\mathrm{PB}}\left(a+r_{c}\right)=\frac{N_{c}}{4 \pi a^{2} \lambda_{G C}} e^{\beta\left(\mu_{c}-\mu_{f}^{\mathrm{ex}}\right)},
$$

This will serve as the renormalized boundary condition for the mPB, Eq. (9), which can now be solved iteratively. For a given $Z$ and $N_{\text {salt }}$, we guess the number of condensed counterions. With this $N_{c}$, we calculate $N_{\text {bulk }}=N_{\text {salt }}-N_{c}$. We then solve the law of mass action Eq. (5) to obtain $N_{p}$ and $N_{f}$. With these values we numerically integrate the $\mathrm{mPB}$ equation to obtain the diffuse density at the surface $\rho_{f}\left(a+r_{c}\right)$. If

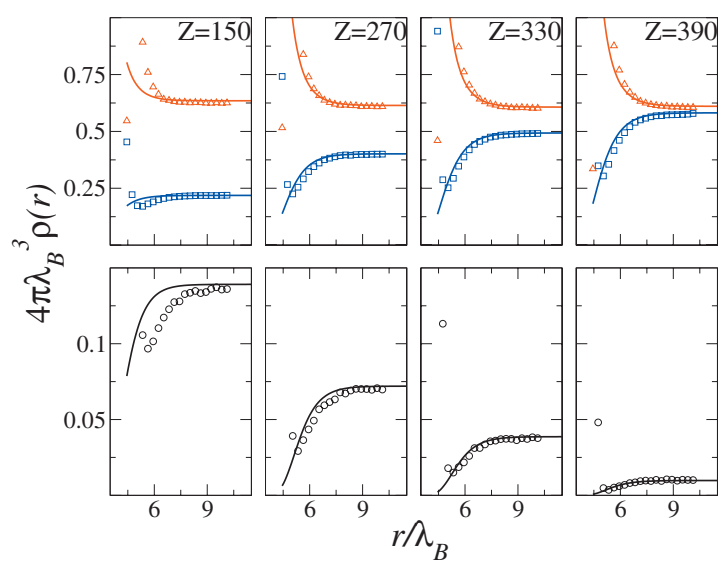

FIG. 2. Density profiles for 3:1 salt $(0.075 \mathrm{M})$ for various colloidal charges. The WS cell radius is $93.05 \AA$. The symbols (same as in Fig. 1) are the simulation data and the lines are obtained with the present theory.

this is not the same as required by Eq. (18), the value of $N_{c}$ is modified, and the procedure is repeated. In practice, the adjustment of $N_{c}$ is performed by coupling the mPB solver to a root finding subroutine, such as the Newton-Raphson, and is very quick.

The total $\alpha$-ion concentration in the bulk is $\rho_{\alpha}(r)$ $=\rho_{f}(r)+\rho_{p}(r)$. In Fig. 2 and Fig. 3, we compare the calculated ionic density profiles with the ones obtained using the MC simulations for various colloidal charges and electrolyte concentrations. The theory is found to describe very accurately the ionic diffuse layer.

The effective charge of the colloid-counterions complex is $Z_{\text {eff }}=Z-\alpha N_{c}$. This is a generalization of the Alexander prescription for suspensions containing multivalent ions. Although the calculation presented here is very different from the one used by Alexander et al., the spirit of the two approaches is the same-charge renormalization allows us to quantitatively calculate the ionic density distribution in the far field using an approximate theory. In Fig. 4, we plot the effective charge versus the bare charge for suspensions containing various concentrations of 3:1 electrolyte. We see that addition of a multivalent electrolyte results in a strong counterion condensation. At sufficiently large concentration of

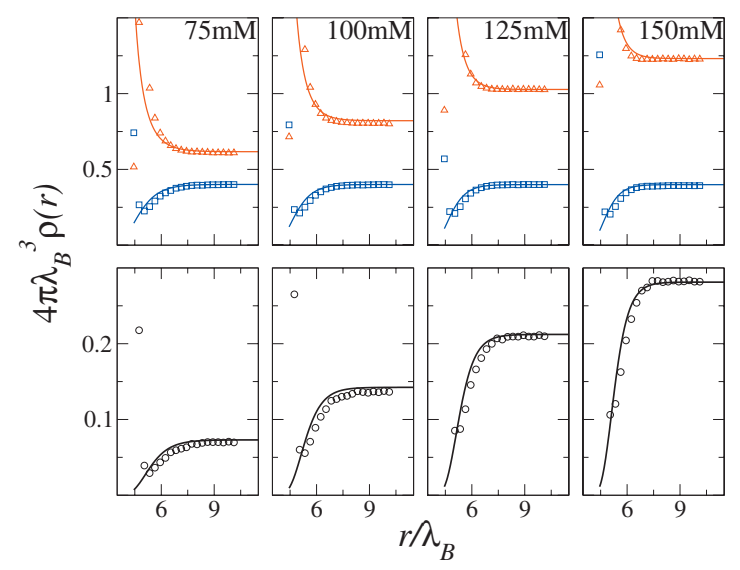

FIG. 3. Density profiles for 3:1 salt for various concentrations. The colloidal charge is $-270 q$ and the WS cell radius is $93.05 \AA$. The symbols (same as in Fig. 1) are the simulation data and the lines are obtained with the present theory. 


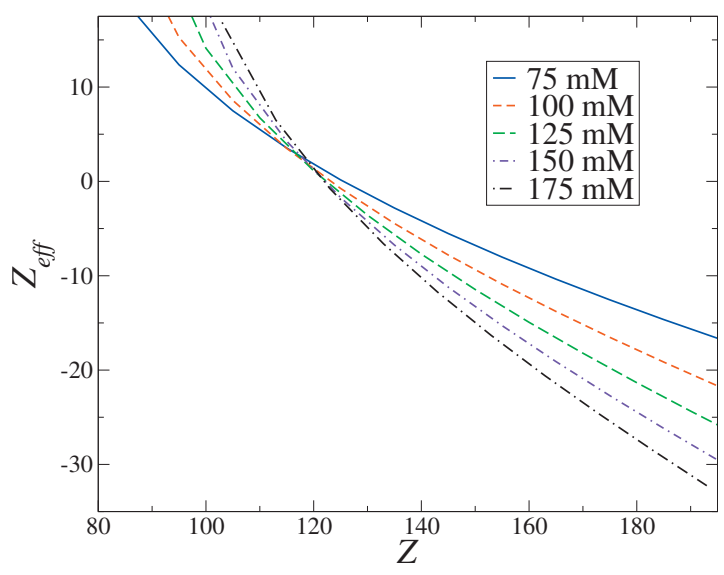

FIG. 4. Effective charges vs colloidal charge, for various 3:1 salt concentrations. The WS cell radius is $93.05 \AA$.

electrolyte, the effective colloidal charge changes sign. $\mathrm{Cu}$ riously, there is a qualitative change in behavior of the renormalized charge between small and high colloidal charges. For small charges, $(Z<120$, for parameters used in this paper), renormalized charge is not a monotonically decreasing function of salt concentration. The reason for this apparently contradictory behavior is that when the colloidal charge is low and there are more coions in the bulk, trivalent ions can gain more free energy by staying there, where their electric field is screened by the cloud of coions. For strongly charged particles $(Z>120)$, addition of electrolyte leads to increased counterion condensation and a uniformly decreasing effective charge.

Since the Alexander prescription cannot be implemented for suspensions containing multivalent counterions, over the years there have been attempts to use a geometric criterion to separate condensed ions from free. ${ }^{9,22,23}$ Unfortunately there is no unique way to set a fixed distance within which ions can be considered to be associated with the colloid. In Fig. 5, we plot the effective colloidal charge that gives the correct diffuse density profile-calculated using the present theory - and compare it with the result of a geometric criterion. We find that to get an optimal agreement, we must set the distance within which ions are considered to be con-
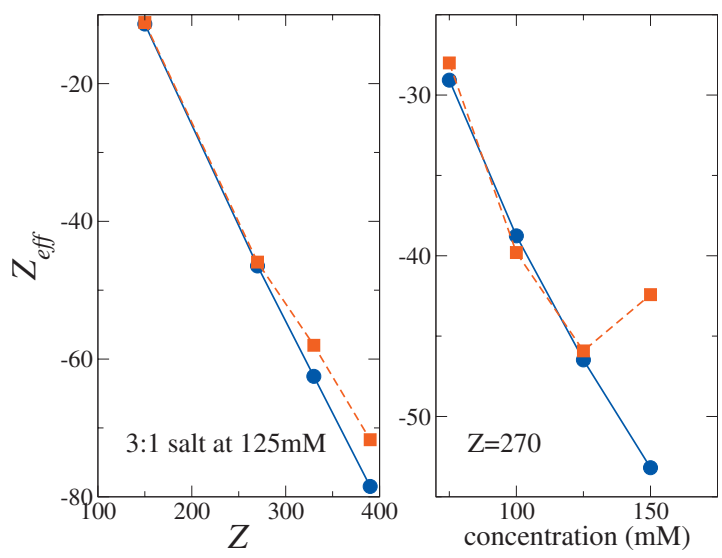

FIG. 5. Effective charges vs colloidal charge and vs 3:1 salt concentration. The circles represents the theory and the squares the geometrical cryterion. The WS cell radius is $93.05 \AA$.

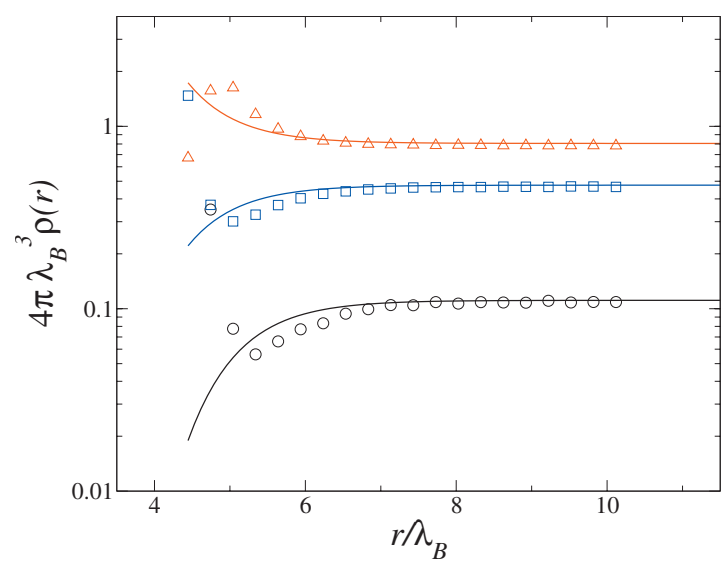

FIG. 6. Density profiles for 3:1 salt (at $80 \mathrm{mM}$ ) and 1:1 salt (at $50 \mathrm{mM}$ ). The colloidal charge is $-225 q$ and the WS cell radius is $93.05 \AA$. The symbols (same as in Fig. 1) are the simulation data and the lines are obtained with the present theory.

densed in simulation to $3.75 \AA$ from the colloidal surfacethat is about one ionic diameter. ${ }^{9}$ However, we see that this distance criterion fails for the strongly charged colloidal particles and for large concentrations of multivalent electrolyte. We conclude that the distance criterions for counterion condensation should be used with some caution. However they can provide a qualitative picture of the counterion condensation.

Over the last few years, there has been a debate about the effect of $1: 1$ salt on the charge reversal. ${ }^{17,24}$ It is quite straightforward to include 1:1 salt in the theory presented here. In Fig. 6, the ionic density profiles are compared with the MC simulations, once again showing a good agreement for the diffuse layer density distributions. In Fig. 7, we plot the effective charge versus the concentration of 1:1 electrolyte at fixed concentration of multivalent salt. For small concentrations of multivalent salt, addition of 1:1 electrolyte hinders the counterion condensation and diminishes the charge reversal. ${ }^{9,17,22}$ At larger concentration of 3:1 electrolyte, small concentrations of $1: 1$ salt can favor slightly the charge reversal. Large concentrations of 1:1 electrolyte are found to always have a negative effect on the overcharging.

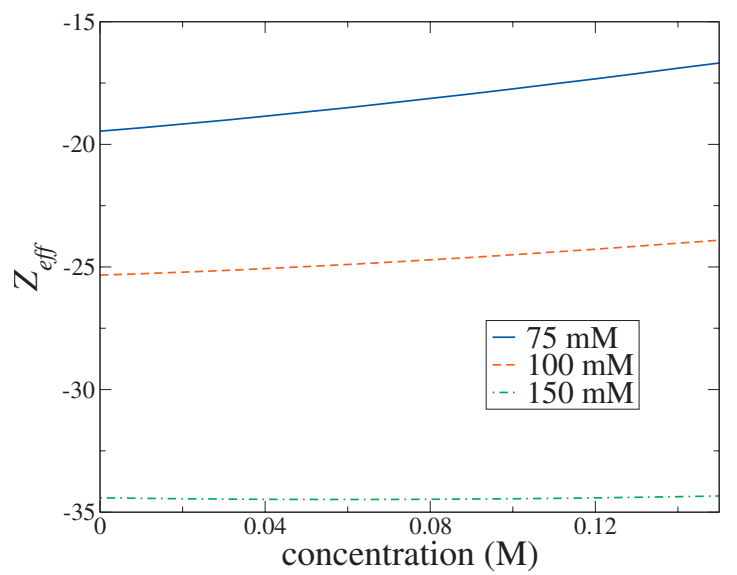

FIG. 7. Effective charges vs 1:1 salt concentration for various $3: 1$ salt concentrations. The colloidal charge is $-210 q$ and the WS cell radius is $93.05 \AA$. 


\section{CONCLUSIONS}

We have presented a theory which allows us to accurately calculate the diffuse layer ionic density profiles in suspensions containing both multivalent and monovalent electrolyte, without any adjustable parameters. The theory also allows us to obtain the number of condensed counterions and to define the effective (renormalized) colloidal charge. The dependence of the effective charge on the concentration of multivalent and the monovalent electrolyte is found to be highly non-trivial. This, to our knowledge, is the only theory that is able to quantitatively describe charge renormalization in colloidal suspensions containing both multivalent and monovalent electrolyte.

\section{ACKNOWLEDGMENTS}

Y.L. would like to acknowledge interesting conversations with Emmanuel Trizac. This work is partially supported by CNPq, INCT-FCx of Brazil, and by the U.S.-AFOSR under the Grant No. FA9550-09-1-0283.

${ }^{1}$ E. Luijten, M. E. Fisher, and A. Z. Panagiotopoulos, Phys. Rev. Lett. 88, 185701 (2002).

${ }^{2}$ Y. Levin, Rep. Prog. Phys. 65, 1577 (2002).

${ }^{3}$ M. Deserno, C. Holm, and S. May, Macromolecules 33, 199 (2000).

${ }^{4}$ I. Rouzina and V. Bloomfield, J. Chem. Phys. 100, 9977 (1996); W. M Gelbart, R. F. Bruinsma, P. A. Pincus, and V. A. Parsegian, Phys. Today 53, 38 (2000); F. J. Solis and M. O. de la Cruz, ibid. 54, 71 (2001).

${ }^{5}$ G. N. Patey, J. Chem. Phys. 72, 5763 (1980); L. Guldbrand, B. Jonsson,
H. Wennerstrom, and P. Linse, ibid. 80, 2221 (1984).

${ }^{6}$ M. Quesada-Pérez, J. Callejas-Fernández, and R. Hidalgo-Álvarez, Adv. Colloid Interface Sci. 95, 295 (2002); A. Fernández-Nieves, A. Fernández-Barbero, F. J. de las Nieves, and B. Vincent, J. Chem. Phys. 123, 054905 (2005).

${ }^{7}$ S. Alexander, P. M. Chaikin, P. Grant, G. J. Morales, P. Pincus, and D. Hone, J. Chem. Phys. 80, 5776 (1984); E. Trizac, L. Bocquet, M. Aubouy, and H. von Grunberg, Langmuir 19, 4027 (2003).

${ }^{8}$ A. Diehl and Y. Levin, J. Phys.: Condens. Matter 17, S3309 (2005).

${ }^{9}$ A. Diehl and Y. Levin, J. Chem. Phys. 125, 054902 (2006).

${ }^{10}$ R. Kjellander and S. Marcelja, J. Phys. Chem. 90, 1230 (1986)

${ }^{11}$ M. Lozada-Cassou, R. Saavedra-Barrera, and D. Henderson, J. Chem. Phys. 77, 5150 (1982).

${ }^{12}$ M. Quesada-Pérez, E. González-Tovar, A. Martín-Molina, M. LozadaCassou, and R. Hidalgo-Álvarez, ChemPhysChem 4, 234 (2003).

${ }^{13}$ R. D. Groot, J. Chem. Phys. 95, 9191 (1991).

${ }^{14}$ M. J. Stevens and M. O. Robbins, Europhys. Lett. 12, 81 (1990).

${ }^{15}$ A. Diehl, M. N. Tamashiro, M. C. Barbosa, and Y. Levin, Physica A 274, 433 (1999).

${ }^{16}$ Y. Levin and M. E. Fisher, Physica A 225, 164 (1996).

${ }^{17}$ S. Pianegonda, M. C. Barbosa, and Y. Levin, Europhys. Lett. 71, 831 (2005).

${ }^{18}$ B. I. Shklovskii, Phys. Rev. E 60, 5802 (1999).

${ }^{19}$ H. Totsuji, J. Phys. Soc. Jpn. 39, 253 (1975).

${ }^{20}$ A. Y. Grosberg, T. T. Nguyen, and B. I. Shklovskii, Rev. Mod. Phys. 74, 329 (2002).

${ }^{21}$ A. P. dos Santos, A. Diehl, and Y. Levin, J. Chem. Phys. 130, 124110 (2009).

${ }^{22}$ O. Lenz and C. Holm, Eur. Phys. J. E 26, 191 (2008).

${ }^{23}$ A. Martín-Molina, C. Rodriguez-Beas, R. Hidalgo-Alvarez, and M. Quesada-Perez, J. Phys. Chem. B 113, 6834 (2009).

${ }^{24}$ T. T. Nguyen, A. Y. Grosberg, and B. I. Shklovskii, Phys. Rev. Lett. 85, 1568 (2000) 